\title{
Calibration of the LAMPF E-960 Polarized Target*
}

\author{
D. A. Hill \\ High Energy Physics Division \\ Argonne National Lab, Argozine, IL 60499
}

December 23, 1992
ANL-HEP-TR--92-111

DE93 007888

\section{Introduction}

\section{OSTI}

This note is mainly about the offline corrections for the target polarization measurements of E-960. For the sake of minimizing the number of separate documents, I have also included my recent thoughts about the target constant, in Sec. 6.

The E-960 data-taking was done in two separate runs. With respect to having an operative NMR system, the runs dated from 27-AUG-87 to 10-DEC-87 and from 14-AUG-88 to 6-OCT-88. These runs will be referred to as the "1987" and "1988" runs, respectively.

Because of the press of intervening projects, I have been unable until now to give my attention to the 1988 run of E-960. The information developed below in Secs. 3-5 is the result of my (belated) attempt to do so. A draft memo dated January 4, 1988 was previously distributed, which concerned the polarization correction factors for the 1987 run. I have reproduced the material from that memo in Sec. 2, for the sake of completeness. Also, since the analysis of the 1988 run has raised some questions about the appropriateness of the older analysis, I have augmented the material from that memo with some of the background observations and calculations that underlay its conclusions, to facilitate a comparison of the two runs.

In Sec. 4 I have made a final synthesis of the observations made during the two runs, and it is there that one will find my best estimate of the offline polarization corrections for both runs.

\section{The 1987 run}

In the 1987 run, all three NMR coils were wrapped around the outside of the target holder. Coil 1 was a hairpin around the downstream end, Coil 2 an upstream hairpin, and Coil 3 a saddle-type around the middle.

- Work supported in part by the U. S. Department of Energy, Division of High Energy Physics, Contract No. W-31-109-ENG-38. 
Table 1: Original multipliers for the 1987 run, from the draft memo.

\begin{tabular}{|c|c|c|c|}
\hline Runs & Multiplier & Runs & Multiplier \\
\hline $424-429$ & $M_{3}=0.795$ & $675-682$ & $M_{2}=1.005$ \\
\hline $430-432 a$ & $M_{3}=0.725$ & $683-707$ & $M_{2}=1.101$ \\
\hline $432 \mathrm{~b}-499$ & $M_{3}=0.804$ & $709-750$ & $M_{2}=0.976$ \\
\hline 546-556 & $M_{1}=0.915$ & $752-813$ & $M_{2}=0.940$ \\
\hline $557-585$ & $M_{3}=0.936$ & $815-842$ & $M_{3}=1.010$ \\
\hline $586-610$ & $M_{3}=0.984$ & $845-854$ & $M_{2}=0.970$ \\
\hline $616-622$ & $M_{2}=1.005$ & $856-869$ & $M_{3}=0.996$ \\
\hline $623-674$ & $M_{3}=1.003$ & $890-906$ & $M_{3}=0.936$ \\
\hline
\end{tabular}

\subsection{Corrections to the thermal equilibrium NMR data}

Table 1 reproduces the list of multipliers from the draft memo for the correction of the online values of the target polarization, $\mathrm{P}_{\mathrm{T}}$, for the 1987 run. These corrections compensate for improper settings of the software parameter $V C A L$, for small offets of the thermal equilibrium (TE) temperatures, for human error, and for drift of the NMR detector. The multiplier subscripts refer to the number of the NMR coil that was the primary monitor for that group of runs. Most of the very large deviations from unity for the initial runs up to 585 are simple software scaling errors caused by mistaken adjustments of $V C A L$ by the operators. Most of the rest of the relatively large deviations are due to NMR drift.

The multipliers of Table 1 should not be used for the final E-960 analysis. See the revised estimates in Sec. 4.

Essentially, a total of 10 sets of thermals were taken during the 1987 run, for a mean interval of $\approx 11$ days between TE's, the largest interval of 18 days falling between runs 707 and 845 . These intervals are generally larger than we would like. For Coils 1 and 3 the $\sigma$ of the distribution of TE-signal areas was 3.6\%; for Coil 2, $4.2 \%$.

\subsection{Coil-comparison polarization data}

After the corrections of Table 1 are applied, global averages of all of the coil comparisons done during the 1987 run give the following results: 


$$
\begin{aligned}
& \text { Summary of all coil comparisons } \\
\overline{\mathrm{P}}_{1}(+)=61.3 \pm 0.6 \%, & \overline{\mathrm{P}}_{1}(-)=-67.8 \pm 0.6 \%, \\
\overline{\mathrm{P}}_{2}(+)=61.3 \pm 0.7 \%, & \overline{\mathrm{P}}_{2}(-)=-69.1 \pm 0.6 \%, \\
\overline{\mathrm{P}}_{3}(+)=59.3 \pm 0.9 \%, & \overline{\mathrm{P}}_{3}(-)=-69.0 \pm 0.5 \% .
\end{aligned}
$$

The errors listed are $1 \sigma$. There is no apparent discrepancy among the coils. The $(-)$ values are significantly higher than the $(+)$ values. Various bits of evidence gathered during the 1987 run suggest fairly strongly that this large asymmetry is due to marginal cooling provisions for this large target. That is, had we been able to apply somewhat higher microwave power, the asymmetry would have lessened.

The sign of the $P_{T}$-values is given in the NMR convention: $(+)$ corresponds io predominant occupation of the Zeeman ground state, i.e., the "thermal" NMRsignals are considered positive. Since the target magnet field pointed downstream, $(+)$ corresponds to target spin parallel to the beam momentum.

\subsection{Original error estimates for $P_{T}$}

After the above corrections, the draft memo gave the estimated total uncertainty on the polarization as $\pm 3.8 \%$, consisting of the following c itributions:
a) Temperature of thermals
$1.0 \%$
b) Statistical uncertaint es of thermals
$1.2 \%$
c) NMR background
$1.0 \%$
d) Spatial uniformity of polarization
$2.5 \%$
e) NMR nonlinearity
$1.0 \%$
f) Residual NMR drift
$2.0 \%$.

It should be stressed that these uncertainties are considered to be " $2 \sigma$ " estimates, i.e., they represent the maximum likely excursions. These contributions are uncorrelated, so they were added in quadrature. Contribution (a) arises primarily from uncertainty in the ${ }^{3} \mathrm{He}$ vapor pressure measurement. Contribution (b) is typical for the $2 \sigma$ errors of the TE's. Contribution (c) is based on past experience with clean target-holder structures made of Teflon. At the time of the 1988 draft memo, contribution (d) was estimated from our prior experience with the E-770 target, since there was no "buried" center coil in the 1987 run. (The E-770 experience suggested that, in this larger target, the polarization in the center could be depressed as much as $5 \%$ relative to the outer regions.) Contribution (e) is known to be typical for NMR detectors of the Liverpool type. Contribution (f) is estimated from the levels of internal consistency of the thermals.

Note that contribution (c) is not a symmetric error; any NMR background is likely to reduce the NMR-measured polarization to a greater extent than the beam- 
Table 2: Thermal-equilibrium-associated corrections for the 1988 run.

\begin{tabular}{ll}
\hline \hline Runs & Multiplier \\
\hline $161-183$ & $M_{2}=0.994$ \\
$186-193$ & $M_{1}=0.941$ \\
$195-203 \mathrm{a}$ & $M_{1}=1.007$ \\
$203 \mathrm{~b}-215$ & $M_{2}=1.003$ \\
$217-221$ & $M_{1}=0.999$ \\
$222-261$ & $M_{2}=0.998$ \\
\hline
\end{tabular}

\begin{tabular}{ll}
\hline \hline Runs & Multiplier \\
\hline $264-279$ & $M_{1}=1.005$ \\
$280-328$ & $M_{1}=0.976$ \\
$329-340$ & $M_{2}=0.990$ \\
$341-368$ & $M_{1}=0.997$ \\
$377-399$ & $M_{2}=1.007$ \\
$400-428$ & $M_{2}=0.998$ \\
\hline
\end{tabular}

sampled polarization. However, since this uncertainty is small, its overall effect is negligible.

The draft memo recommended that the total uncertainty be increased to $\pm 5 \%$ for runs 424-499 iscause of NMR instability. Again, see Sec. 4 for my final estimate of the errors.

\section{The 1988 run}

For the 1988 run, Coil 3 was changed to a hairpin "buried" at the center of the target. Coils 1 and 2 were unchanged.

\subsection{Corrections to the thermal equilibrium NMR data}

As before, I have generated a list of multipliers to correct for errors detectable in the online NMR records. For the sake of completeness, these TE-associated corrections are listed here in Table 2, but they should not be employed directly for the scattering data analysis, for the reason that I describe further on. Again, the multiplier subscripts refer to the number of the NMR coil. The corrections are small, except for the two Coil 1-data intervals in which the thermal signal areas differed significantly before and after the interval. (There appears to be no obvious connection between whatever happened during these two intervals and the problems to be noted below. For example, the Coil 2-TE areas changed very little during these intervals, so they do not seem to mark the occurrence of any "global glitches.")

Essentially, a total of 14 sets of thermals were taken during the run, for a mean interval of $\approx 4$ days between TE's, with the largest interval of 8 days falling between runs 340 and 377 . For Coil 1 the $\sigma$ of the distribution of TE-signal areas was $6.1 \%$; for Coil 2, 1.5\%; and for Coil 3,1.8\%. For some reason, Coil 1 was considerably less reliable than Coil 2 in 1988. 


\subsection{Coil-comparison polarization data}

During the 1988 run, it was soon noticed that Coil 1 (outer downstream hairpin) polarization measurements were consistently lower than Coil 2 (outer upstream hairpin) measurements by several percentage points. Unfortunately, owing to technical problems, reliable data from the "buried" Coil 3 were not available until the last week of the run. When Coil 3 was finally enabled, it was found to give values even higher than those of Coil 2, by several percentage points. These discrepancies remain after the offline TE-associated corrections are made, as shown in the following summaries of corrected global averages:

$$
\begin{array}{ll} 
& \text { Summary of all coil comparisons } \\
\overline{\mathrm{P}}_{1}(+)=58.7 \pm 0.5 \%, & \overline{\mathrm{P}}_{1}(-)=-64.0 \pm 0.6 \%, \\
\overline{\mathrm{P}}_{2}(+)=62.9 \pm 0.4 \%, & \overline{\mathrm{P}}_{2}(-)=-67.1 \pm 0.8 \% \\
\overline{\mathrm{P}}_{3}(+)=63.5 \pm 1.3 \%, & \overline{\mathrm{P}}_{3}(-)=-70.4 \pm 0.8 \% .
\end{array}
$$

This summary includes all comparisons that were made, a large number of which involved only Coils 1 and 2 in the earlier parts of the run. A more valid relationship is presumably obtained by limiting the data to the direct comparisons, among all the coils, made late in the run:

Summary of coil comparisons with three coils measured

$$
\begin{array}{ll}
\overline{\mathrm{P}}_{1}(+)=56.1 \pm 0.4 \%, & \overline{\mathrm{P}}_{1}(-)=-62.9 \pm 0.1 \%, \\
\overline{\mathrm{P}}_{2}(+)=60.7 \pm 0.7 \%, & \overline{\mathrm{P}}_{2}(-)=-66.8 \pm 0.5 \%, \\
\overline{\mathrm{P}}_{3}(+)=63.5 \pm 1.3 \%, & \overline{\mathrm{P}}_{3}(-)=-70.4 \pm 0.8 \% .
\end{array}
$$

The errors are $1 \sigma$. It is clear that significant differences existed among all three coils, for each sign of polarization. The $(-)$ values are significantly higher than the $(+)$ values, as was true in the 1987 run, and this was presumably for the same reason (target oversized relative to the cooling provisions). In 1987, however, a significant difference was not seen between Coils 1 and 2 .

There are a variety of possible causes for such discrepancies:

1. Spatially nonuniform magnetic field.

2. Spatially nonuniform microwave field.

3. Differing NMR backgrounds.

4. Spatially nonuniform beads, e.g., beads marginally overheated during target insertion.

5. Spatially nonuniform refrigeration. 
Other internal evidence does not favor causes (1) or (2). Cause (3) would require that the three coils were "dirty" in differing degrees. This cannot be ruled out, but normally adequate cleaning procedures were apparently followed, so it seems unlikely to be the culprit. Cause (4) also cannot be ruled out, but there is nothing in the record that hints at any temperature excursion large enough. This leaves us with cause (5), and, indeed, this was the cause proposed by the LAMPF personnel during the run (PT Log, p. 237). For both runs of this target, the FEP Teflon target holder (perforated all around) was surrounded, with a few mm of radial clearance, by the TFE Teflon $\mathrm{L}^{3} \mathrm{He}$ container, which had openings only at the top to allow vapor to escape. It was felt that the topmost part of the beads might not be in liquid (since there may have to be some interior vapor space) and, therefore, some of the beads sensed by the outer hairpins (and by the saddle coil in the 1987 run) might not be well cooled. On the other hand, all of the "buried" center coil would be well below the hypothetical vapor space. The different relationship between Coils 1 and 2 in the two runs could be due to slight differences in the target holder conformation and angle relative to gravitational vertical, for example. All in all, this seems to be the most plausible scenario for the observed nonuniformity.

\section{Final conclusions for $\mathrm{P}_{\mathrm{T}}$}

\subsection{Adjusted multipliers for the 1988 data}

The above hypothesis for the origin of the 1988 nonuniformity would imply the existence of a relatively thin layer of poorly polarized beads at the top of the target, for both runs. Thus, it might well be that the Coil 3 measurements of 1988 are more representative of the beam-sampled polarization than are those of the other coils. Nevertheless, since that cause cannot be positively confirmed, I think that the best approach is to adjust the $1988 \mathrm{P}_{\mathrm{T}}$-values, effectively, to reflect a simple arithmetic average of the three coils and to cover our ignorance by expanding the errors accordingly.

To that end, the above three-coil comparisons yield the following ratios

$$
\begin{aligned}
& \left\langle\overline{\mathrm{P}}_{i}(+)\right\rangle / \overline{\mathrm{P}}_{1}(+)=60.1 / 56.1=1.071 \\
& \left\langle\overline{\mathrm{P}}_{i}(-)\right\rangle / \overline{\mathrm{P}}_{1}(-)=66.7 / 62.9=1.060 \\
& \left\langle\overline{\mathrm{P}}_{i}(+)\right\rangle / \overline{\mathrm{P}}_{2}(+)=60.1 / 60.7=0.990 \\
& \left\langle\overline{\mathrm{P}}_{i}(-)\right\rangle / \overline{\mathrm{P}}_{2}(-)=66.7 / 66.8=0.999
\end{aligned}
$$

where the averages are straight averages over the three coils $i=1,2,3$. Since the $(+,-)$ ratios for each coil do not differ significantly, we can simplify things by combining the $(+,-)$ data: 
Table 3: Complete multipliers for the 1988 run.

\begin{tabular}{lcc}
\hline \hline Runs & Coil & $\begin{array}{c}\text { Multiplier } \\
R_{i} M_{i}\end{array}$ \\
\hline $161-183$ & 2 & 0.989 \\
$186-193$ & 1 & 1.002 \\
$195-203 \mathrm{a}$ & 1 & 1.073 \\
$203 \mathrm{~b}-215$ & 2 & 0.997 \\
$217-221$ & 1 & 1.064 \\
$222-261$ & 2 & 0.992 \\
\hline
\end{tabular}

\begin{tabular}{lcc}
\hline \hline Runs & Coil & $\begin{array}{c}\text { Multiplier } \\
R_{\boldsymbol{i}} M_{\boldsymbol{i}}\end{array}$ \\
\hline $264-279$ & 1 & 1.071 \\
$280-328$ & 1 & 1.040 \\
$329-340$ & 2 & 0.985 \\
$341-368$ & 1 & 1.062 \\
$377-399$ & 2 & 1.002 \\
$400-428$ & 2 & 0.993 \\
\hline \hline
\end{tabular}

$$
\begin{aligned}
& R_{1}=\left\langle\left|\overline{\mathrm{P}}_{i}\right|\right\rangle /\left\langle\left|\overline{\mathrm{P}}_{1}\right|\right\rangle=63.40 / 59.50=1.066 \\
& R_{2}=\left\langle\left|\overline{\mathrm{P}}_{i}\right|\right\rangle /\left\langle\left|\overline{\mathrm{P}}_{2}\right|\right\rangle=63.40 / 63.75=0.995
\end{aligned}
$$

where the $R_{i}$ are now to be combined with the $M_{i}$ of Sec. 3.1 to give the complete offline multipliers. This is done in Table 3. These are the multipliers that should be used in the scattering data analysis for the 1988 run.

For the later purpose of forming the error estimates (see Secs. 4.3,4), I note that $\left\langle\left|\bar{P}_{3}\right|\right\rangle /\left\langle\left|\bar{P}_{i}\right|\right\rangle=66.95 / 63.40=1.056$.

\subsection{Adjusted multipliers for the 1987 data}

If we are to apply the above considerations in a consistent way, the 1987 data should also be adjusted to reflect the (in this case hypothetical and unmeasured) higher polarization at the target center. This can be done with the average of the ratios of Sec. 4.1, $R=\left(R_{1}+R_{2}\right) / 2=1.030$, applied to each of the three coils in Table 1. The result is listed in Table 4. These are the multipliers that should be used in the E-960 analysis for the 1987 run.

\subsection{Error estimates for $P_{T}$ for the 1988 run}

After the corrections of Sec. 4.1, the estimated total uncertainty on the polarization is $\pm 6.1 \%$, consisting of the following " $2 \sigma$ " contributions:
a) Temperature of thermals
$1.0 \%$
b) Statistical uncertainties of thermals $1.4 \%$
c) NMR background $\quad 1.0 \%$
d) Spatial uniformity of polarization $\quad 5.6 \%$
e) NMR n onlinearity
$1.0 \%$
f) Residual NMR drift
$0.8 \%$. 
Table 4: Complete multipliers for the 1987 run.

\begin{tabular}{|c|c|c|c|c|c|}
\hline Runs & Coil & $\begin{array}{c}\text { Multiplier } \\
\qquad R M_{i}\end{array}$ & Runs & Coil & $\begin{array}{c}\text { Multiplier } \\
R M_{i}\end{array}$ \\
\hline $424-429$ & 3 & 0.819 & $675-682$ & 2 & 1.035 \\
\hline $430-432 a$ & 3 & 0.747 & $683-707$ & 2 & 1.134 \\
\hline $432 b-499$ & 3 & 0.828 & $709-750$ & 2 & 1.005 \\
\hline $546-556$ & 1 & 0.942 & $752-813$ & 2 & 0.968 \\
\hline $557-585$ & 3 & 0.964 & $815-842$ & 3 & 1.040 \\
\hline $586-610$ & 3 & 1.014 & 845-854 & 2 & 0.999 \\
\hline $616-622$ & 2 & 1.035 & $856-869$ & 3 & 1.025 \\
\hline $623-674$ & 3 & 1.033 & 890-906 & 3 & 0.964 \\
\hline
\end{tabular}

These contributions have been estimated in ways similar to those of Sec. 2.3, except that contribution (d) is derived from the observed ratio $\left\langle\left|\overline{\mathbf{P}}_{3}\right|\right\rangle /\left\langle\left|\overline{\mathbf{P}}_{i}\right|\right\rangle$ of Sec. 4.1. Note that, if the likely scenario for the observed nonuniformity as discussed in Sec. 3.2 is true, then contribution (d) is not symmetric. Since this is the dominant error here, the total uncertainty may be highly skewed.

\subsection{Adjusted error estimates for $P_{T}$ for the 1987 run}

In the spirit of the above, the 1987 error estimate (Sec. 2.3) should now be:
a) Temperature of thermals
$1.0 \%$
b) Statistical uncertainties of thermals
$1.2 \%$
c) NMR background
$1.0 \%$
d) Spatial uniformity of polarization $\quad 5.6 \%$
e) NMR nonlinearity
$1.0 \%$
f) Residual NMR drift
$2.0 \%$,

that is, the estimate for contribution (d) is increased to the 1988 level. The total " $2 \sigma$ " uncertainty is $\pm 6.3 \%$.

\section{Observation of a trend in the peak polarization in 1988}

In the process of performing these analyses, I have noticed what appears to be a trend toward lower $\mathrm{P}_{\mathrm{T}}$-values over the course of the 1988 run. This is illustrated in Fig. 1, where the peak polarization is plotted versus days of running. The multipliers 


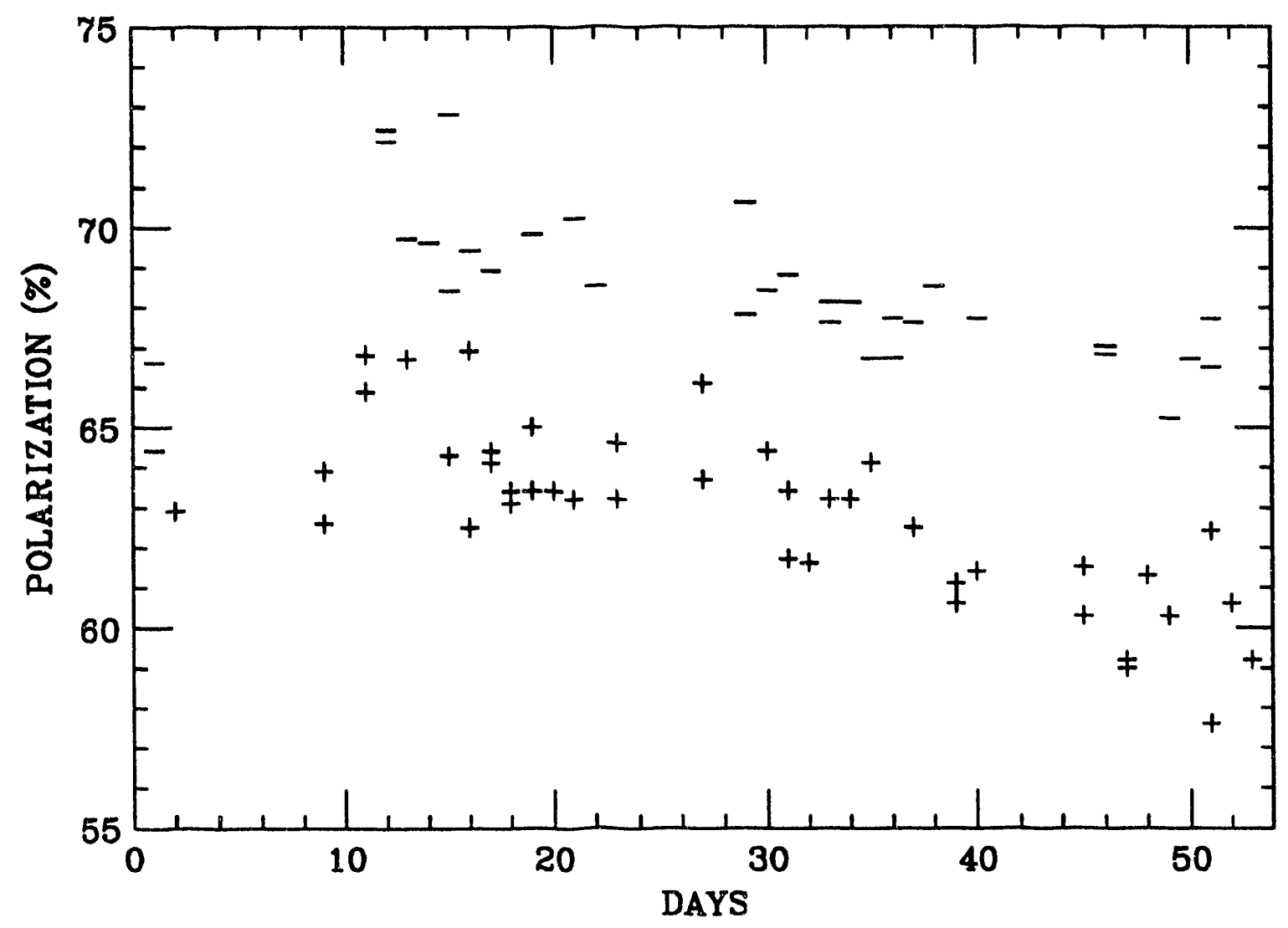

Figure 1: Maximum attained $\mathrm{P}_{\mathrm{T}}$-values. Positive values are plotted as $(+)$, negative as $(-)$. Day 1 is 15 -AUG-88.

of Table 3 have been applied. (Only results of measurements on Coils 1 and 2 are plotted, since the Coil 3 data span very little cime.) Aside from the sparse data in the first nine days, when the "shakedown" of target operations was still occurring, both signs of $\mathrm{P}_{\mathrm{T}}$ seem to show a gradual decrease with time, on the average.

The only situation in which such behavior is normally seen is with the use of intense beams, leading to cumulative radiation damage. Since this apparently cannot be the case for E-960, I am at a loss for a plausible explanation. One might seek a connection between this puzzle and the above nonuniformity problem. For example, if the amount of ${ }^{3} \mathrm{He}$ in the target were decreasing over time then the $\mathrm{P}_{\mathrm{T}}$ values might follow this trend. However, according to the PT Log all of the gas was recovered at the end of the run. Moreover, there were three occasions during the run (on days 4,8 , and 46 of Fig. 1) when additional ${ }^{3} \mathrm{He}$ from the Auxiliary Tank was injected into the loop, and the polarization showed little immediate response.

Another possibility is that the refrigerator was slowly "plugging" progressively during the run. There is no evidence for this in the pressure vs flow data recorded 


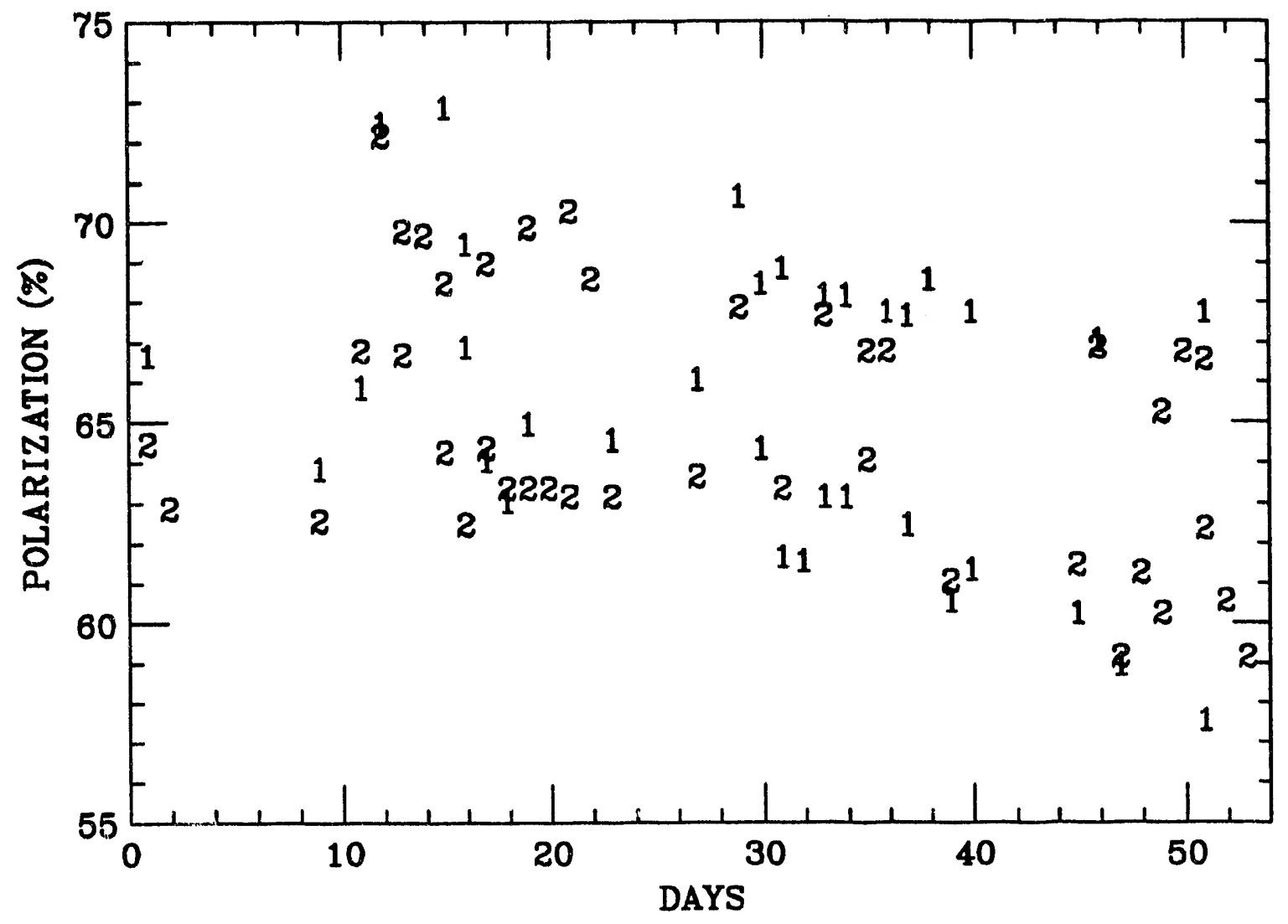

Figure 2: Maximum attained $\mathrm{P}_{\mathrm{T}}$-values. The plot is identical to that of Fig. 1 except that the symbols correspond to the number of the coil from which the data were derived.

over the run. There is, in the Log, recorded evidence that there was a tendency to apply slightly more conservative (lower) microwave power as the run progressed. This may explain part of the trend, but it seems unable to explain all of it.

For what it's worth, I have seplotted the data of Fig. 1 in Fig. 2, replacing the plot symbols with the coil numbers from which the data originated. It seems that both coils show approximately the same trend, within the scatter.

It is worth noting that this trend, whatever the cause, shows up in a subtle way in the coil comparison summaries of Sec. 3.2. It is mainly this trend that leads to somewhat lower values for $\left|\bar{P}_{1,2}\right|$ in the three-coil summary than in the all-comparison summary, because all of the three-coil data came late in the run.

The $P_{T}$-data from 1987 do not exhibit any significant trend vs running time. 


\section{The target constant}

Recently, John Jarmer made a series of measurements of the outer dimensions of the E-960 target holder. With allowance for the thickness of the FEP, the results for the warm internal dimensions were: diameter $D=4.75 \pm 0.10 \mathrm{~cm}$, length $L=$ $12.54 \pm 0.08 \mathrm{~cm}$, and the uncertainties are $1 \sigma$. The cold $(T \leq 77 \mathrm{~K})$ dimensions are inferred using a thermal shrinkage of $1.7 \%$ for FEP Teflon:

$$
\begin{aligned}
& D(\text { cold })=4.67 \pm 0.11 \mathrm{~cm} \\
& L(\text { cold })=12.33 \pm 0.13 \mathrm{~cm}
\end{aligned}
$$

where the estimated uncertainty has been increased by adding half of the shrinkage in quadrature. The volume enclosed by the holder is thus $V($ cold $)=211 \pm 12 \mathrm{~cm}^{3}$. The uncertainty here is conservative (overestimated), since I have assumed correlated errors, whereas only the shrinkage part of the errors should be correlated.

The target material was a mixture of 1,2-propanediol and EHBA-Crv in the following proportions:

$$
\begin{array}{lr}
\mathrm{C}_{3} \mathrm{H}_{8} \mathrm{O}_{2} & 96.46 \text { wt\% } \\
\mathrm{C}_{12} \mathrm{H}_{2} \mathrm{CrNaO}_{8} & 3.54 \text { wt\% }
\end{array}
$$

which corresponds to an overall elemental composition of

$$
\begin{array}{lr}
\mathrm{C} & 47.06 \text { wt\% } \\
\mathrm{O} & 41.79 \text { wt\% } \\
\mathrm{H} & 10.43 \text { wt\% } \\
\mathrm{Cr} & 0.50 \text { wt\% } \\
\mathrm{Na} & 0.22 \text { wt\%. }
\end{array}
$$

I consider that the uncertainties on these numbers should be much less than $1 \%$. However, chemical analyses were done on the target materials from both E-960 runs, with the following reported results:

$$
\begin{array}{llr}
\text { Target } 1(1987): & \text { C } & 46.70 w t \% \\
& \text { H } & 9.95 \text { wt\% } \\
\text { Target 2(1988): } & \text { H } & 10.63 \text { wt } \% \text {. }
\end{array}
$$

(Carbon analysis was not reported for the second target.) I have no completely satisfactory explanation for the large difference between the reported H-contents. My chemist contacts assure me that a "well done" elemental analysis, for materia!s like this, should be accurate to $1 \%$. (That is, $1 \%$ of a $10 \mathrm{wt} \%$ value, or $0.1 \mathrm{wt} \%$.) Perhaps these analyses were not so "well done"? In some of the more trustworthy analytical reports that $I$ have seen, the individual values for a series of analytical runs 
are listed, and then a group average is obtained and the statistical errors estimated from the scatter. In such listings it is not unusual to see individual values that differ from the mean by $0.2 \mathrm{wt} \%$ or more. The above reports list only a single value with no error estimate.

At the end of buth runs, the target material was recovered and weighed, with the following results:

$$
\begin{array}{ll}
\text { Target } 1 \text { mass } & 154.2 \pm 0.1 \mathrm{~g} \\
\text { Target } 2 \text { mass } & 137.2 \pm 1.4 \mathrm{~g} \text {. }
\end{array}
$$

The same target holder was used for both runs, with the exception that the \#3 NMR coil was buried in the target in 1988. This "coil" consisted of a 20.3-cm length of $\mathrm{Cu} / \mathrm{Ni}$ tubing covered with $0.127-\mathrm{cm}-\mathrm{O}$.D. Teflon spaghetti and folded into a hairpin with a width of 0.4-0.5 in. Bead packing effects increase the effective diameter of the spaghetti to $\approx 0153 \mathrm{~cm}$. Thus, the effective volume occupied by the buried coil was $\approx 0.4 \mathrm{~cm}^{3}$, wh not large enough to explain the big difference in measured mass. The density of this standard material is rather well known: $\rho($ cold $)=1.14 \pm 0.02 \mathrm{~g} / \mathrm{cm}^{3}$. If we assume a canonical packing fraction of 0.64 , the expected mass of target material would thus be

$$
\begin{aligned}
M(\text { expected }) & =0.64 \rho(\text { cold }) V(\text { cold }) \\
& =0.64(1.14 \pm 0.02)(211 \pm 12) \\
& =154 \pm 9 \mathrm{~g} .
\end{aligned}
$$

While, in a superficial sense, both measured masses are within the expected range, they must be considered to differ significantly, since the uncertainty here mainly represents our ignorance of the true (fixed) volume of the holder. Therefore, I believe that the 1988 target must have had a significant $\left(\approx 10 \mathrm{~cm}^{3}\right)$ void, probably located at the top of the holder. Since very little of the beam would be expected to traverse such a void, I believe that the best approach is to assume that both targets had the same effective density, that of the 1987 target.

Considering all of the above, I estimate the target constants $A_{1,2}$ as follows. Let $N_{\mathrm{H}}$ be the number of hydrogens in the target and let $a$ be the cross-sectional area of the target. Then the target constant $A_{1}$ for the 1987 run is

$$
\begin{aligned}
A_{1} & =a / N_{\mathrm{H}} \\
& =\pi D^{2} / 4\left[f M_{1}\left(6.022 \times 10^{23}\right) / 1.008\right] \\
& =1315 D^{2} / f M_{1} \\
& =1315(4.67 \pm 0.12)^{2} /(0.1043 \pm 0.0034)(154.2 \pm 0.1) \\
& =1783 \pm 109 \mathrm{mb},
\end{aligned}
$$

where $f$ is the weight fraction of hydrogen and the uncertainty is $1 \sigma$. I have increased the uncertainty on $D$ hy enough to allow (in quadrature) for a $2 \%$ void in Target 1. 
("Boundary" packing effects are negligible for such a large diameter target.) For $f$ I have used the value for the nonginal composition and assigned an uncertainty equal to half of the difference between the reported analytical results. For $A_{2}$, the only difference is that the error is expanded (in quadrature) by half of the difference between $M_{1}$ and $M_{2}$, in order to account for the possibility that the 1988 void was not all at the top of the holder:

$$
A_{2}=1783 \pm 147 \mathrm{mb} .
$$

\title{
DISCLAIMER
}

\begin{abstract}
This report was prepared as an account of work sponsored by an agency of the United States Government. Neither the United States Government nor any agency thereof, nor any of their employees, makes any warranty, express or implied, or assumes any legal liability or responsibility for the accuracy, completeness, or usefulness of any information, apparatus, product, or process disclosed, or represents that its use would not infringe privately owned rights. Reference herein to any specific commercial product, process, or service by trade name, trademark, manufacturet, or otherwise does not necessarily constitute or imply its endorsement, recommendation, or favoring by the United States Government or any agency thereof. The views and opinions of authors expressed herein do not necessarily state or reflect those of the United States Government or any agency thereof.
\end{abstract}



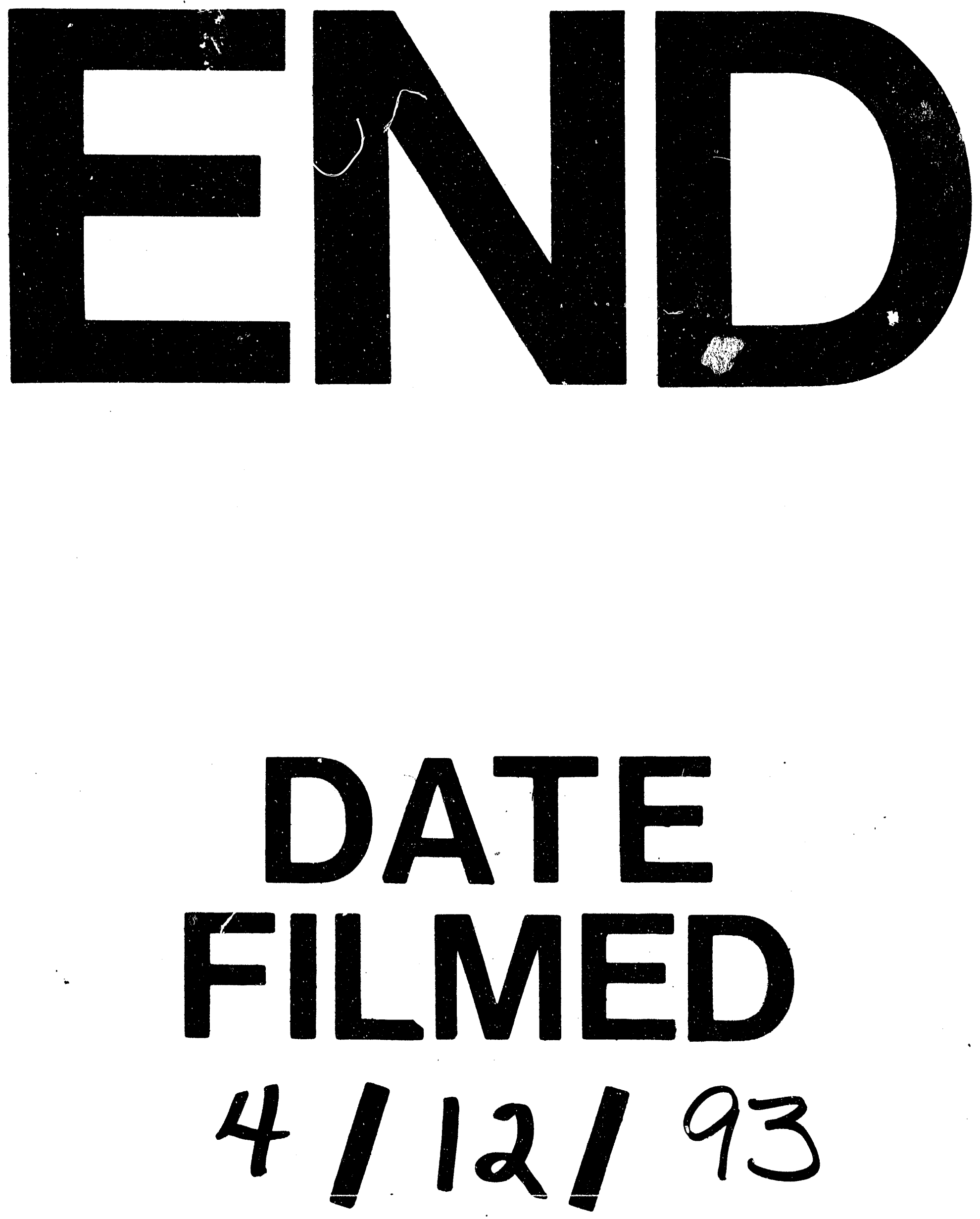
